\title{
Phase transitions and exchange interactions in the $\mathrm{SmCr}_{3}\left(\mathrm{BO}_{3}\right)_{4}$ crystal
}

\author{
E.A. Dobretsova ${ }^{1,2, *}$, K.N. Boldyrev ${ }^{1}$, M.N. Popova ${ }^{1}$, S.Yu. Gavrilkin ${ }^{2}$, A.A. Mukhin ${ }^{3}$, \\ V.Yu. Ivanov' ${ }^{3}$, V.V. Mal'tsev ${ }^{4}$, N.I. Leonyuk ${ }^{4}$, and B.Z. Malkin ${ }^{5}$ \\ ${ }^{1}$ Institute for Spectroscopy RAS, 108480 Troitsk, Moscow, Russia \\ ${ }^{2}$ P.N. Lebedev Physical Institute RAS, 119991 Moscow, Russia \\ ${ }^{3}$ A.M. Prokhorov General Physics Institute RAS, 119991 Moscow, Russia \\ ${ }^{4}$ M.V. Lomonosov Moscow State University, 119991 Moscow, Russia \\ ${ }^{5}$ Kazan Federal University, 420008 Kazan, Russia
}

\begin{abstract}
Spectroscopic investigation and specific heat and magnetic susceptibility measurements of $\mathrm{SmCr}_{3}\left(\mathrm{BO}_{3}\right)_{4}$ crystals were performed. The spectra of the $\mathrm{Sm}^{3+}$ and $\mathrm{Cr}^{3+}$ ions in samarium chromium borate were calculated and parameters of the exchange interactions between the nearest chromium ions, chromium and samarium ions were determined. Three phase transitions were observed at the temperatures $T_{1}=7.8 \pm 0.5 \mathrm{~K}, T_{2}=6.7 \pm$ $0.5 \mathrm{~K}$, and $T_{3}=4.3 \pm 0.2 \mathrm{~K}$, their nature is discussed. The crystal structures with different space symmetry groups $R 32$ and $C 2 / c$ were found to coexist in a $\mathrm{SmCr}_{3}\left(\mathrm{BO}_{3}\right)_{4}$ single crystal.
\end{abstract}

Samarium chromium borate $\mathrm{SmCr}_{3}\left(\mathrm{BO}_{3}\right)_{4}$ is a member of a large family of rare-earth (RE) borates with structural type of the natural mineral huntite. These compounds are described by a general formula $R M_{3}\left(\mathrm{BO}_{3}\right)_{4}$, where $R$ is $\mathrm{Y}$ or $\mathrm{La}-\mathrm{Lu}$ and $M$ is $\mathrm{Al}, \mathrm{Ga}, \mathrm{Fe}, \mathrm{Cr}$, or Sc. The compounds are actively investigated due to their interesting physical properties and a promising application potential. However, RE chromium borates are not so well studied. It is known that $\mathrm{NdCr}_{3}\left(\mathrm{BO}_{3}\right)_{4}$ is an easy-plane antiferromagnet below the temperature $8.0 \mathrm{~K}$ [1] and that $\mathrm{EuCr}_{3}\left(\mathrm{BO}_{3}\right)_{4}$ undergoes an antiferromagnetic phase transition at $T_{\mathrm{N}} \approx 9 \mathrm{~K}$ [2]. A phase transition at $5 \mathrm{~K}$ was supposed to occur in $\mathrm{SmCr}_{3}\left(\mathrm{BO}_{3}\right)_{4}$ [3].

We succeeded to prepare a big oriented single crystal of $\mathrm{SmCr}_{3}\left(\mathrm{BO}_{3}\right)_{4}$ and to perform magnetic measurements on it. It follows from the temperature dependences of the magnetic susceptibility that the magnetic moments of chromium and samarium ions (samarium ions are polarized by the exchange field) are ordered in the $a b$-plane. However, as this sample was not preserved, heat capacity and optical spectra measurements were carried out on other samples. Crystal characterization by X-ray diffraction and infrared (IR) spectroscopy revealed a polytypic nature of $\mathrm{SmCr}_{3}\left(\mathrm{BO}_{3}\right)_{4}$. The crystals can be rhombohedral (space group R32) or monoclinic $(C 2 / c)$, depending on growth conditions. Moreover, domains of both structural phases can coexist in one single crystal. For measurements we chose the crystals with a predominant content of the $R 32$ phase. The temperature dependence of the

* Corresponding author: elenadobretsova89@gmail.com 
heat capacity $C(T)$ of $\mathrm{SmCr}_{3}\left(\mathrm{BO}_{3}\right)_{4}$ has revealed two second-order phase transitions, at $T_{1}=$ $7.8 \mathrm{~K}$ and $T_{2}=6.7 \mathrm{~K}$, and the first-order transition at $T_{3}=4.3 \mathrm{~K}$.

Optical spectra registered in the range of $f-f$ transitions in the $\mathrm{Sm}^{3+}$ ions and $d-d$ transitions in the $\mathrm{Cr}^{3+}$ ions helped to identify energy level positions in these ions in the crystal field $(\mathrm{CF})$ of $\mathrm{SmCr}_{3}\left(\mathrm{BO}_{3}\right)_{4}$. It was also found from the observed spectral lines splitting that the compound orders magnetically at a temperature near $7 \mathrm{~K}$.

The set of experimental data allowed determining CF parameters for the $\mathrm{Sm}^{3+}$ and $\mathrm{Cr}^{3+}$ ions in $\mathrm{SmCr}_{3}\left(\mathrm{BO}_{3}\right)_{4}$. The $g$-factors, calculated in this paper for the ground orbital singlet of $\mathrm{Cr}^{3+}$, are $g_{a a}=1.9648, g_{b b}=1.9675, g_{c c}=1.9702$ but the initial CF splitting parameter is $D=-$ $0.379 \mathrm{~cm}^{-1}$. When considering magnetic properties of $\mathrm{SmCr}_{3}\left(\mathrm{BO}_{3}\right)_{4}$, we supposed the presence of isotropic exchange interactions between the nearest neighbor chromium and samarium ions $\left(H_{f d}=-2 J_{f d} \boldsymbol{S}_{C r} \boldsymbol{S}_{S m}\right)$, chromium ions inside the chains $\left(H_{12}=-2 J_{n n} \boldsymbol{S}_{C r 1} \boldsymbol{S}_{C r 2}\right)$, and the next nearest neighbor chromium ions in the closest chains $\left(H_{12}^{\prime}=-2 J_{n n n} \boldsymbol{S}_{C r 1} \boldsymbol{S}_{C r 2}\right)$. A projection of the exchange interaction operator of the $\mathrm{Sm}^{3+}$ ion with one of the closest $\mathrm{Cr}^{3+}$ ions onto the ground state (the lowest doublet) of the samarium ion in the crystal-field leads to a spin Hamiltonian

$$
H_{f d}^{(e f f)}=-2 J_{f d}\left[G_{\perp}\left(S_{C r, x} S_{x}+S_{C r, y} S_{y}\right)+G_{\|} S_{C r, z} S_{z}\right]
$$

where $S_{\alpha}$ are components of the effective spin of the samarium ion $S=1 / 2$, and $G_{\|}=0.2743$, $G_{\perp}=2.2$.

Temperature dependences of the static magnetic susceptibility tensor components and magnetic-field dependences of the magnetization were calculated in the self-consistent field approximation, using a model of clusters (dimers) containing the closest chromium ions in a chain. This model is analogous to the model of dimers in the chains of iron ions, used formerly to investigate magnetic and spectral properties of the RE iron borates [4]. A comparison between the calculated and experimental temperature dependences of the magnetic susceptibility gave the values $J_{n n}=-2.8 \mathrm{~K}$ and $J_{n n n}=0.75 \mathrm{~K}$ for the exchange integrals; the value $J_{f d}=0.132 \mathrm{~K}$ was obtained from the measured samarium ion ground-state splitting in the exchange field at low temperatures on the assumption of a collinear antiferromagnetic structure in the chains of chromium ions.

It follows from the analysis of magnetic characteristics of $\mathrm{Sm}^{3+}$ and $\mathrm{Cr}^{3+}$ that a hypothesis about a spin-reorientational nature of the low-temperature transition with a rotation of magnetic moments from the $a b$ plane to the $c$ axis is not valid. The main question about the exchange interaction parameters in comparison with iron borates refers to the exchange interaction between the chromium chains. According to neutron scattering data, the iron ions are ferromagnetically ordered in every $a b$ plane, due to the antiferromagnetic interaction $\left(J_{n n n}<0\right)$ of the closest chains. Because of the change of sign in the exchange integral $J_{n n n}$ in $\mathrm{SmCr}_{3}\left(\mathrm{BO}_{3}\right)_{4}$, one may expect a magnetic structure essentially different from the one observed in iron borates. Additional investigation is required to determine a magnetic structure of chromium borates.

This work was supported by the Russian Science Foundation (Grant No 14-12-01033). E.A.D. acknowledges a Scholarship of the President of the Russian Federation (SP754.2015.1).

\section{References}

1. E.A. Popova et al., Phys. Rev. B 75, 054446 (2007)

2. L. Gondek et al., J. Sol. St. Chem. 210, 30 (2014)

3. K.N. Boldyrev, E.P. Chukalina, N.I. Leonyuk, Phys. Sol. St. 50, 1681 (2008)

4. M.N. Popova et al., Phys. Rev. B 75, 224435 (2007) 